Monatsschrift Kinderheilkd 2005 · 153:360-363 DOI 10.1007/s00112-003-0773-3

Online publiziert: 4. September 2003

(c) Springer Medizin Verlag 2003

\section{Redaktion}

D. Reinhardt, München

\author{
M. Janner · P. E. Mullis \\ Abteilung für Pädiatrische Endokrinologie und Diabetologie, \\ Medizinische Universitätskinderklinik Bern
}

\title{
Osteopenie und pathologische Frakturen bei einem Jugend- lichen mit Laktoseintoleranz und hohem Oxalatkonsum
}

gen. Skleren weiß. Internistisch unauffällig. Die Pubertätsentwicklung war altersentsprechend: $\mathrm{P} 4$, Hodenvolumen $15 \mathrm{ml}$ beidseits.

\section{Diagnostik}

Die Knochendensitometrie (DEXA mit Hologic QDR450o W) zeigte folgende Werte: LWS (L2-L4) o,581 g/ $\mathrm{cm}^{2}, \mathrm{z}-\mathrm{ScO}-$ re $-2,1$; Schenkelhals $0,769 \mathrm{~g} / \mathrm{cm}^{2}, \mathrm{z}-\mathrm{ScO}$ re $-0,5$.

Das Knochenalter nach Greulich und Pyle betrug 14 Jahre.

Das biochemische Bild bei Erstvorstellung war mit einem sekundären Hyperparathyreoidismus vereinbar. Differenzialdiagnostisch konnte mittels Screening der Eltern, die beide ein normales Serumkalzium, normales iPTH und eine normale Kalziurie aufwiesen, eine familiäre hypokalziurische Hyperkalzämie ausgeschlossen werden (• Tabelle 1 ).

Wir beurteilten das klinische Bild dieses Jugendlichen als Osteoporose im Rahmen eines sekundären Hyperparathyreoidismus bei kalziumarmer Ernährung und begannen dem entsprechend einen Therapieversuch mit $1 \mathrm{~g} \mathrm{CaCO}_{3}$-Zusatz/Tag.

\section{Verlauf}

Während der darauf folgenden 10 Monate blieb das iPTH unverändert hoch. Da der Patient aber schließlich zugab, den Kalzi- umzusatz kaum je eingenommen zu haben, sahen wir uns nicht zu weiterer Diagnostik veranlasst. Erst 10 Monate nach Erstvorstellung begann der Patient, die Medikation zuverlässig einzunehmen. Dies wird in den Laboruntersuchungen durch die Zunahme der Kalziurie auf o,23 mmol/ mmol Kreatinin dokumentiert.

Ein halbes Jahr später (d. h. 16 Monate nach Erstvorstellung) zeigte die Knochendensitometrie in der Tat eine Zunahme der Knochendichte an allen Messstellen:

\footnotetext{
- LWS (L2-L4) o,748 g/cm², z-score -o,8; Zunahme: $28,7 \%$,

- Schenkelhals $0,850 \mathrm{~g} / \mathrm{cm}^{2}$, z-score -o,1; Zunahme 10,5\%.
}

Die Körperlänge entwickelte sich zwischen den beiden Knochendensitometrien im gleichen Perzentilenkanal: Anlässlich der ersten Messung betrug der Längen-SDS 1,7, 16 Monate danach 1,76. Bei vergleichbarem Längen-SDS hatte somit die Flächenknochendichte jedenfalls im Bereich der LWS deutlich zugenommen. Es ist wahrscheinlich, dass dies als normale Zunahme der Knochenmasse im Rahmen der Pubertät anzusehen ist.

Überraschenderweise fand sich allerdings eine weitere Erhöhung des iPTHWertes bei normalen Kalzium- und Phosphatwerten und niedriger Kalziurie. Das gleichzeitig erhöhte $1,25(\mathrm{OH})_{2}$-Vitamin- 
$\mathrm{D}_{3}$ interpretierten wir als Ausdruck einer gesteigerten 1-a-Hydroxylase-Aktivität im Rahmen des Hyperparathyreoidismus. Klinisch war der Patient unauffällig, Wachstum und Pubertätsentwicklung waren ebenfalls normal.

Wegen der Persistenz des Hyperparathyreoidismus wurde eine erweiterte Abklärung durchgeführt. Die differenzialdiagnostischen Überlegungen, die uns dabei leiteten, sind aus $\bullet$ Tabelle 2 ersichtlich.

Die weiterführenden Abklärungen ergaben folgende Resultate:

- Hb 136 g/l (normal 136-168), Ec $5,13 \times 10^{12} / 1, \mathrm{MCV} 83 \mathrm{fl}$, red cell distribution width (RDW) 17\% (normal 11,514,5), Leukozyten 5,4. Ferritin 12 mcrg/ 1 (normal 10-30o). Normal waren: $\mathrm{Na}$, $\mathrm{K}, \mathrm{Cl}$, Osmolalität, Albumin, Kreatinin, Harnstoff, Vitamin A, Vitamin E, Quick, venöse Blutgasanalyse, Urin$\mathrm{pH}$ und fraktionierte Ausscheidung von $\mathrm{Na}, \mathrm{K}, \mathrm{Cl}$, Glukose, Protein im Urinspot. Die Zöliakieserologie war bei normalem Gesamt-IgA unauffällig.

- Schilddrüsensonographie: Kein Anhalt für Nebenschilddrüsenadenom.

Wegen der anamnestischen Angaben des Meidens von Milchprodukten seit frühester Kindheit wurde eine Laktosebelastung durchgeführt. Diese zeigte eine erhöhte $\mathrm{H}_{2}$-Ausscheidung im Atemtest, die mit einer Laktoseintoleranz vereinbar war.

Nachdem andere Ursachen für einen Hyperparathyreodismus ausgeschlossen worden waren, interpretierten wir daher den sekundären Hyperparthyreoidismus des Jugendlichen als Folge einer Kalziummalabsorption im Rahmen einer Laktoseintoleranz. Ein weiterer Hinweis auf eine leichtgradige Malabsorption war das Hämoglobin im unteren Normbereich bei erhöhter RDW und Ferritin im untersten Referenzbereich. Verstärkend kam bei diesem Patienten wahrscheinleich der hohe Oxalatkonsum in der Form von IceTee (200-300 mg/Tag) hinzu. Dementsprechend führten wir eine Diätberatung durch und begannen eine strikte laktosefreie und oxalatarme Diät. Ein halbes Jahr später hatte sich, wie aus der letzten Spalte in - Tabelle 1 ersichtlich ist, der PTHWert normalisiert. Das Hämoglobin war auf $153 \mathrm{~g} / \mathrm{l}$ und die Erythrozytenzahl auf $5,4 \times 10^{12} / 1$ angestiegen.

\section{Diskussion}

Wir beschreiben in diesem Bericht einen Jugendlichen mit Osteopenie mit rezidivierenden pathologischen Frakturen im Rahmen eines sekundären Hyperparathyreoidismus bei sehr niedriger alimentärer Kalziumzufuhr im Kontext einer Laktoseintoleranz und zusätzlich hoher Oxalateinnahme.

Aus der Literatur ist die Beziehung zwischen Laktosemalabsorption und Osteopenie/Osteoporose bekannt: Bei postmenopausalen Frauen ist Laktosemalabsorption zwar nicht per se mit verminderter BMD (bone mineral density) assoziert, aber wenn die Gruppe nach Vorhandensein von Symptomen für Laktoseintoleranz stratifiziert wird, zeigen die laktoseintoleranten Frauen eine signifikant verminderte BMD bei gleichzeitig reduzierter alimentärer Kalziumzufuhr [1]. Eine finnische Studie bei prä- und perimenopausalen Frauen konnte ein deutlich erhöhtes Frakturrisiko bei Frauen mit Laktoseintoleranz zeigen [2]. Auch bei Kindern konnte gezeigt werden, dass eine laktosearme Diät durch Meiden von Milchprodukten wegen verminderter Kalziumzufuhr zu einer inadäquaten Knochenmineralisation führt [3]. Eine kürzlich publizierte Untersuchung bei jungen Erwachsenen [4] zeigte ferner, dass Individuen mit Laktosemalabsorption, die Intoleranzsymptome hatten, eine um etwa $30 \%$ niedrigere alimentäre Kalziumzufuhr hatten und eine signfikant niedrigere BMD an Lendenwirbelsäule und Schenkelhals aufwiesen. Bei diesen Patienten war das iPTH im oberen Normbereich.

Diese Daten aus der Literatur lassen den Schluss zu, dass laktoseintolerante Patienten, wegen der nach deren Verzehr auftretenden Symtpome, Milchprodukte meiden und wegen der damit verbundenen langjährigen schlechteren Kalziumzufuhr eine Verminderung der Knochendichte aufweisen. Diese ist mit einem erhöhten Frakturrisiko verbunden. Unseres Wissens wurde allerdings bis anhin noch kein Kind oder Jugendlicher mit solch einer Problematik in der Literatur beschrieben. Wir glauben, dass bei unse-
Monatsschrift Kinderheilkd 2005 $153: 360-363$ DOI 10.1007/s00112-003-0773-3

(c) Springer Medizin Verlag 2003

M. Janner · P. E. Mullis

Osteopenie und pathologische Frakturen bei einem Jugendlichen mit Laktoseintoleranz und hohem Oxalatkonsum

\section{Zusammenfassung}

Wir stellen einen Jugendlichen mit Osteopenie und pathologischen Frakturen vor. Der Fall soll auf folgende Punkte aufmerksam machen:

Eine adäquate Kalziumzufuhr während Kindheit und Adoleszenz ist für die Entwicklung einer normalen „peak bone mass" von ausschlaggebender Bedeutung. Es ist wahrscheinlich, dass moderne Ernährungsgewohnheiten (Junkfood und Softdrinks) bisher noch zu wenig erkannte Auswirkungen auf die Gesundheit unserer Knochen haben.

\section{Schlüsselwörter}

Osteopenie - Osteoporose ·

Sekundärer Hyperparathyreoidismus · Oxalat · Junkfood · Softdrinks

Osteonpenia and pathological fractures in an adolescent with lactose intolerance and high oxalate intake

\section{Abstract}

We report an adolescent with osteopenia and pathologic fractures. This case should draw the attention on the following points. An adequate calcium supply during childhood and adolescence is important for the development of normal peak bone mass. Modern eating habits with high intake of junk food and soft drinks are likely to have important impact on bone health.

\section{Keywords}

Osteopenia - Osteoporosis .

Secondary hyperparathyroidism .

Oxalic acid · Junk food · Soft drinks 
Tabelle 1

Laborwerte bei Erstvorstellung und den folgenden Monaten unter Therapie

\begin{tabular}{|c|c|c|c|c|c|c|}
\hline & Referenzwerte & $\begin{array}{l}\text { Erst- } \\
\text { vorstellung }\end{array}$ & $\begin{array}{l}\text { Nach } \\
4 \text { Monaten }\end{array}$ & $\begin{array}{l}\text { Nach } \\
10 \text { Monaten }\end{array}$ & $\begin{array}{l}\text { Nach } \\
16 \text { Monaten }\end{array}$ & $\begin{array}{l}\text { Nach } \\
23 \text { Monaten }\end{array}$ \\
\hline Ca tot & $2,1-2,6 \mathrm{mmol} / \mathrm{l}$ & 2,45 & 2,5 & 2,5 & 2,31 & 2,42 \\
\hline Ca ion & $1,13-1,3 \mathrm{mmol} / \mathrm{l}$ & 1,2 & 1,24 & 1,33 & 1,19 & 1,23 \\
\hline Phosphat & $1-2,0 \mathrm{mmol} / \mathrm{l}$ & 1,58 & 1,52 & 1,69 & 1,53 & 1,33 \\
\hline Magnesium & $0,73-1,05 \mathrm{mmol} / \mathrm{l}$ & 0,8 & & & & \\
\hline Albumin & $30-50 \mathrm{~g} / \mathrm{l}$ & 42 & 43 & & 38 & 42 \\
\hline AP & $108-300 \mathrm{U} / \mathrm{I}$ & 600 & 584 & & 355 & \\
\hline BSAP & ${ }^{\mathrm{a}} \mathrm{U} / \mathrm{I}$ & 569 & & & & \\
\hline IGF-1 & $184-581 \mathrm{ng} / \mathrm{ml}$ & 360 & & & & \\
\hline $25 D$ & $6-40 \mathrm{ng} / \mathrm{ml}$ & 29,7 & 25 & 15,8 & 12,0 & \\
\hline $1,25 \mathrm{D}$ & $30-85 \mathrm{pg} / \mathrm{ml}$ & 127 & 118 & 192,4 & 125 & \\
\hline iPTH & $10-65 \mathrm{pg} / \mathrm{ml}$ & 86 & 93 & 94 & 113 & 33 \\
\hline Kreatinin & 25-106 mcrmol/l & 74 & 74 & & 81 & 86 \\
\hline U:DPD & $\mathrm{mmol} / \mathrm{mmol} \mathrm{Kreat}$ & 27,5 & & & & \\
\hline UCa/UKr & $\mathrm{mmol} / \mathrm{mmol}$ Kreat & 0,07 & 0,08 & 0,23 & 0,07 & 0,15 \\
\hline
\end{tabular}

a Keine Referenzwerte für Kinder bekannt; Ca tot Kalzium total, Ca ion ionisiertes Kalzium, AP alkalische Phosphatase, BSAP Knochenisoenzym der alkalischen Phosphatase, 25D 25(OH) Vitamin-D3, 1.25D 1.25(OH) 2 Vitamin-D3, iPTH intakt Parathormon, U:DPD Desoxypyrridinolin im Urin, UCa/UKr Urin Kalzium-Kreatinin-Verhältnis

Tabelle2

Differenzialdiagnose des primären und sekundären Hyperparathyreoidismus

\begin{tabular}{|c|c|c|}
\hline Lokalisation & Diagnose & Diagnostik \\
\hline \multirow[t]{4}{*}{ Intestinal } & Malabsorptionssyndrom (z. B. Zöliakie) & Blutbild, Ferritin, Quick, Zäliakieserologie Vitamin A, E \\
\hline & Laktoseintoleranz & $\mathrm{H}_{2}$-Atemtest \\
\hline & Alimentär stark verminderte Ca-Zufuhr & - \\
\hline & Vitamin-D-Mangel & 25-OH-Vitamin-D3 \\
\hline \multirow[t]{2}{*}{ Renal } & Niereninsuffizienz & Harnstoff, Kreatinin, Albumin, $\mathrm{Na}, \mathrm{K}$ \\
\hline & Renotubuläre Azidose & BGA, Urinspot: $\mathrm{Na}$, K, Glukose, Protein, pH, Kreat \\
\hline Endorgandefekt & Pseudohypoparathyreoidismus & a \\
\hline Ca-sensing Rezeptor & $\mathrm{FHH}^{\mathrm{b}}$ & b \\
\hline Nebenschilddrüse & Beginnender primärer Hyperparathyreoidismus bei Adenom & Sonographie \\
\hline
\end{tabular}

rem Patienten verschärfend die übermäßige Oxalatzufuhr hinzukam. Es ist gut bekannt, dass Oxalat die intestinale Kalziumabsorption durch Bildung unlöslicher Kalziumoxalatkomplexe reduziert $[5,6]$. Allerdings wurde die Frage der Auswirkungen eines starken Ice-Tee-Konsums auf den Kalziumstoffwechsel bei westlichen Jugendlichen bisher nicht gezielt untersucht. Etwas widersprüchlich scheint auf den ersten Blick die Tatsache, dass unser Indexpatient auch bei genauem Befragen über keine Intoleranzsymptome klagte. Wir denken allerdings, dass dies darauf zurückzuführen ist, dass er bereits seit frühester Kindheit konsequent Milchprodukte mied und somit gar nicht genügend Laktose aufnahm, um Intoleranzsymptome zu entwickeln.

Zusammenfassend glauben wir, dass dieser Fallbericht folgende zwei Punkte mit Eindrücklichkeit zeigt:
1. Die Bedeutung einer genügenden Kalziumzufuhr während Kindheit und Adoleszenz für die Entwicklung einer normalen „peak bone mass“.

2. Es ist wahrscheinlich, dass moderne Ernährungsgewohnheiten (Stichwort "Junkfood“ und „Softddrinks") bisher noch zu wenig erkannte Auswirkungen auf die Gesundheit unserer Knochen haben. 


\section{Korrespondierender Autor Dr. M. Janner}

Abteilung für Pädiatrische Endokrinologie und Diabetologie,

Medizinische Universitätskinderklinik,

CH-3010 Bern

E-Mail: marco.janner@bluewin.ch

\section{Danksagung}

Wir danken Herrn Professor Dr. F. Frey, Chefarzt der Abteilung für Nephrologie und Hypertonie des Inselspitals Bern, ganz herzlich für die Mitarbeit in der Betreuung dieses Patienten.

\section{Literatur}

1. Corazza GR, Benati G, Di Sario A, Tarozzi C, Strocchi A, Passeri M, Gasbarrini G (1995) Lactose intolerance and bone mass in postmenopausal Italian women. Br J Nutr 73:479-487

2. Honkanen R, Kroger H, Alhava E, Turpeinen P, Tuppurainen M, Saarikoski $S$ (1997) Lactose intolerance associated with fractures of weight-bearing bones in Finnish women aged 38-57 years. Bone 21:473-477

3. Infante $D$, Torno $R$ (2000) Risk of inadeguate bone mineralization in diseases involving long-term suppression of dairy products. J Pediatr Gastroenterol Nutr 30:310-313

4. Di Stefano M, Veneto G, Malserviti S, Cecchetti L, Minguzzi L, Strocchi A, Corazza GR (2002) Lactose malbsorption and intolerance and peak bone mass. Gastroenterology 122:1793-1799

5. Heaney RP, Weaver CM (1989) Oxalate:effect on calcium absorbability. Am J Clin Nutr Oct 50(4):830832

6. Weaver CM, Martin BR, Ebner JS, Krueger CA (1987) Oxalic acid decreases calcium absorption in rats. J Nutr Nov 117(11):1903-1906

\section{Das neue Gesicht von Nuklein- säuren}

Alarmsignal für das Immunsystem

Seit einiger Zeit wird eine bestimmte Form von kurzen Nukleinsäure-Stückchen, so genannte kurze interferierende Ribonukleinsäure (short interfering RNA, siRNA) eingesetzt, um die Bildung beliebiger Proteine in der Zelle ganz gezielt abzuschalten (RNA-Interferenz). Diese Technik hat die Möglichkeiten zur Entschlüsselung des menschlichen Erbguts revolutioniert.

Gunther Hartmann, Veit Hornung und weitere Mitarbeitern der Universität München gelang es nun die Interaktion von siRNA mit dem Immunsystem aufzuklären. Die bisher vorherrschende Meinung war, dass siRNA zu klein ist, um durch das Immunsystem wahrgenommen zu werden, bzw. um zu einer Aktivierung des Immunsystems zu führen. Nun konnte hingegen eine hochspezialisierte Zelle des Immunsystems identifiziert werden, die tatsächlich in der Lage ist, siRNA effektiv zu erkennen. Dabei handelt es sich um die sog. plasmazytoide dendritische Zelle. Diese detektiert ganz spezifisch Viren und aktiviert daraufhin das Immunsystem zur Ausschüttung von Interferon. Die Münchner Wissenschaftler haben entdeckt, dass die Erkennung von siRNA durch das Immunsystem von der Sequenzabfolge der Nukleinsäure abhängt, und über einen auf diesen Vorgang spezialisierten Rezeptor erfolgt - den Toll-like receptor 7 . Weiterhin wurden hierbei bestimmte Sequenzmotive identifiziert, die das Immunsystem in einen besonders effektiven Alarmzustand versetzen, wie dies bei einer tatsächlich vorliegenden Virusinfektion der Fall ist. Aus der Entdeckung ergeben sich 3 wichtige Konsequenzen:

- ein neuer Mechanismus ist aufgeklärt, über den das Immunsystem Nukleinsäuren, wie sie in Viren vorkommen, erkennt und diese abwehrt,

- beim therapeutischen Einsatz von siRNA zur gezielten Hemmung von krankmachenden Proteinen könnten ganz gezielt immunologische Sequenzmotive der siRNA eliminiert oder als zusätzliche Wirkkomponenten in diese eingesetzt werden und

- es kann nun siRNA generiert werden, mit der das Immunsystem ausschließlich und gezielt gegen Virusinfektionen gesteuert wird.

Die Ergebnisse wurden in Nature Medicine publiziert (Hornung et al., Vol. 11, März 2005).

Quelle: Klinikum der Universität München, www.klinikum.uni-muenchen.de 